\title{
On the temporal evolution of the stellar mass function of Galactic clusters
}

\author{
Guido De Marchi, ${ }^{1}$ Francesco Paresce ${ }^{2}$ and Simon Portegies Zwart ${ }^{3}$ \\ ${ }^{1}$ ESA, Space Science Department, Keplerlaan 1, 2200 AG Noordwijk, the Netherlands \\ email: gdemarchi@rssd.esa.int \\ ${ }^{2}$ INAF - IASF, Via Gobetti 101, 40129 Bologna, Italy \\ ${ }^{3}$ Sterrewacht Leiden, Leiden University, Postbus 9513, 2300 RA Leiden, the Netherlands
}

\begin{abstract}
We show that we can obtain a good fit to the present-day stellar-mass functions of a large sample of young and old Galactic clusters with a tapered Salpeter power-law distribution function with an exponential truncation of the form $\mathrm{d} N / \mathrm{d} m \propto m^{\alpha}\left[1-\exp \left(-m / m_{\mathrm{c}}\right)^{\beta}\right]$. The average value of the power-law index $\alpha$ is $\sim-2.2$, very close to the Salpeter value of -2.3 , while the characteristic mass, $m_{\mathrm{c}}$, is in the range $0.1-0.6 \mathrm{M}_{\odot}$ and does not seem to vary in any systematic way with the present cluster parameters such as metal abundance, total cluster mass or central concentration. However, the characteristic mass shows a remarkable correlation with the dynamical age of the cluster, namely $m_{\mathrm{c}} / \mathrm{M}_{\odot} \simeq 0.15+0.5 \times t_{\mathrm{dyn}}^{3 / 4}$, where $t_{\mathrm{dyn}}$ is the dynamical time, taken as the ratio of cluster age and dissolution time. The small scatter around this correlation is likely due to uncertainties on the estimated value of $t_{\mathrm{dyn}}$. We attribute the observed trend to the onset of mass segregation through two-body relaxation in a tidal environment, causing preferential loss of low-mass stars from the cluster and hence a drift of the characteristic mass towards higher values. If dynamical evolution is indeed at the origin of the observed trend, it seems plausible that globular clusters, now with $m_{\mathrm{c}} \simeq 0.35 \mathrm{M}_{\odot}$, were born with a stellar mass function very similar to that measured today in the youngest Galactic clusters and with a value of $m_{\mathrm{c}}$ around $0.15 \mathrm{M}_{\odot}$. This is consistent with the absence of a turn-over in the mass function of the Galactic bulge down to the observational limit at $\sim 0.2 \mathrm{M}_{\odot}$ and argues for the universality of the initial mass function of Population I and II stars.
\end{abstract}

Keywords. globular clusters: general, open clusters and associations: general, stars: luminosity function, mass function

\section{Introduction}

As stated in the scientific rationale of this Symposium, there is general consensus that stars do not form in isolation but rather from the fragmentation of molecular clouds, leading to star cluster formation (e.g., Elmegreen, these proceedings). This makes stellar clusters ideal places to study the properties of star formation and its end result, the stellar initial mass function (IMF). However, it is also equally well established today that the vast majority of stars, in our Galaxy and elsewhere, are not in clusters but in the field, since clusters disrupt over time (see, e.g., Gieles, these proceedings). Therefore, any attempt to set constraints on the star-formation mechanisms from the analysis of the present-day stellar mass function $(\mathrm{MF})$ of but the youngest clusters cannot ignore the consequences of their dynamical evolution.

This issue becomes particularly important if we want to compare the results of star formation in physically different environments that are noncoeval. The obvious example is addressing differences in the way low-mass stars $\left(<1 \mathrm{M}_{\odot}\right)$ formed in globular clusters (GCs), at $z \simeq 5$, and in young clusters in the local Universe. While in both cases the raw data show a broad plateau in the mass distribution, suggesting a characteristic mass on 
the order a few tenths of $\mathrm{M}_{\odot}$ (e.g., Elmegreen et al. 2008), until the effects of dynamical evolution are properly understood no conclusion can be drawn as to the uniformity of the star-formation process across time, i.e., on the universality of the IMF.

On the other hand, even though the two-body relaxation process that governs the cluster's dynamical evolution and leads to preferential loss of low-mass stars from the cluster is today rather well understood (e.g., Spitzer 1987), it is in general not possible to roll back the effects of dynamics and derive the IMF from the present-day MF of a cluster, since we cannot trace back the trajectories of stars that have escaped from the cluster. It is, however, possible and statistically meaningful to study the evolution of the stellar MF on a global scale by looking at the differences between clusters at different evolutionary stages. This requires a homogeneous sample of high-quality observations of Galactic (open and globular) clusters, treated in a uniform way and with reliable errors. Early in this decade, this type of homogeneous study became possible for GCs (Paresce \& De Marchi 2000), mostly thanks to the Hubble Space Telescope (HST). Meanwhile, highquality data have become available for young clusters (YCs) as well, mostly from widefield ground-based surveys, thereby making this study possible on a global scale. We present here the preliminary results of this work.

\section{The tapered power law}

Since our goal is to detect and quantify changes in the shape of the MF in different environments, in particular in GCs and YCs, we need a functional form for the distribution of stellar masses that is flexible enough to adapt to the variety of observed MFs, yet simple enough to be described by a small number of parameters over a wide mass range. Kroupa (2002) proposed a segmented, multipart power law for this purpose, but we cannot adopt it here because this approach fixes the mass points between which the slope is fitted, thereby making it impractical or even impossible to detect variations in the characteristic mass which, as we will see, are the signature of dynamical evolution.

Our choice falls instead on a tapered power-law (TPL) distribution of the type

$$
f(m)=\frac{\mathrm{d} N}{\mathrm{~d} m} \propto m^{\alpha}\left[1-e^{\left(-m / m_{c}\right)^{\beta}}\right]
$$

where $m_{\mathrm{c}}$ is the characteristic mass, $\alpha$ the index of the power-law portion for high masses and $\beta$ the tapering exponent that defines the shape of the MF below $m_{c}$. The TPL combines in one expression the ubiquitous power-law shape above $1 \mathrm{M}_{\odot}$ (e.g., Salpeter 1955) with the plateau and drop observed near the hydrogen-burning limit (e.g., Chabrier 2003). As we already showed (De Marchi et al. 2005), the TPL fits remarkably well the MFs of both YCs and GCs over the entire stellar mass range. We stress here that it is irrelevant whether the IMF resulting from star formation takes on the shape of a TPL, as long as it suitably represents the observed mass distribution.

\section{The data sample}

The data used in this work come from a number of different sources. As regards GCs, we consider the entire HST sample of Paresce \& De Marchi (2000), which consists of twelve relatively dense clusters, and add to it four low-concentration GCs studied by our team in recent years (see Table 1). As De Marchi et al. (2007) showed, a strong trend exists between a cluster's concentration and the shape of its present MF, so it is important to cover both dense and loose objects. As for the YCs, the data cover 
Table 1. The sample of YCs, dense and loose GCs used in this study.

\begin{tabular}{|c|c|c|c|c|c|c|c|c|c|c|c|c|c|}
\hline Name & $\alpha$ & $\beta$ & $m_{\mathrm{c}}$ & $\log t$ & $t_{\mathrm{dyn}}$ & Ref & Name & $\alpha$ & $\beta$ & $m_{\mathrm{c}}$ & $\log t$ & $t_{\mathrm{dyn}}$ & Ref \\
\hline$\rho \mathrm{Oph}$ & -1.8 & 1.8 & 0.15 & 5.7 & 0.001 & I & Orion & -2.2 & 2.4 & 0.20 & 6.0 & 0.002 & $\mathrm{G}, \mathrm{M}$ \\
\hline Taurus & -2.3 & 2.1 & 0.33 & 6.0 & 0.25 & $\mathrm{H}$ & IC 348 & -2.0 & 3.0 & 0.14 & 6.3 & 0.004 & $\mathrm{~K}$ \\
\hline$\sigma$ Ori & -2.0 & 2.6 & 0.18 & 6.5 & 0.006 & D & $\lambda$ Ori & -1.8 & 2.4 & 0.10 & 6.7 & 0.01 & A \\
\hline Chamaeleon 1 & -2.1 & 2.0 & 0.22 & 6.7 & 0.01 & $\mathrm{H}$ & IC 2391 & -2.1 & 2.6 & 0.16 & 7.7 & 0.06 & B \\
\hline Blanco 1 & -1.7 & 2.0 & 0.20 & 8.1 & 0.09 & $\mathrm{~J}$ & Pleiades & -2.2 & 2.3 & 0.27 & 8.1 & 0.06 & $\mathrm{~J}$ \\
\hline M 35 & -1.8 & 2.4 & 0.33 & 8.2 & 0.08 & B & Coma Ber & -1.3 & 1.7 & 0.16 & 8.6 & 0.48 & G \\
\hline Praesepe & -2.0 & 3.5 & 0.26 & 8.8 & 0.18 & $\mathrm{E}$ & Hyades & -2.1 & 2.8 & 0.45 & 8.8 & 0.37 & $\mathrm{C}$ \\
\hline NGC 104 & -2.3 & 2.4 & 0.35 & 10.1 & 0.15 & $\mathrm{~L}$ & NGC 5139 & -2.3 & 2.7 & 0.35 & 10.1 & 0.23 & L \\
\hline NGC 5272 & -2.3 & 2.9 & 0.35 & 10.1 & 0.15 & $\mathrm{~L}$ & NGC 6121 & -2.3 & 3.1 & 0.37 & 10.1 & 0.89 & $\mathrm{~L}$ \\
\hline NGC 6254 & -2.3 & 2.4 & 0.35 & 10.1 & 0.52 & $\mathrm{~L}$ & NGC 6341 & -2.3 & 2.3 & 0.33 & 10.1 & 0.50 & $\mathrm{~L}$ \\
\hline NGC 6397 & -2.3 & 2.7 & 0.35 & 10.1 & 0.68 & $\mathrm{~L}$ & NGC 6656 & -2.3 & 2.7 & 0.33 & 10.1 & 0.31 & $\mathrm{~L}$ \\
\hline NGC 6752 & -2.3 & 2.9 & 0.37 & 10.1 & 0.40 & $\mathrm{~L}$ & NGC 6809 & -2.3 & 2.5 & 0.33 & 10.1 & 0.61 & $\mathrm{~L}$ \\
\hline NGC 7078 & -2.3 & 2.7 & 0.28 & 10.1 & 0.15 & $\mathrm{~L}$ & NGC 7099 & -2.3 & 2.2 & 0.29 & 10.1 & 0.52 & $\mathrm{~L}$ \\
\hline NGC 2298 & -2.3 & 3.5 & 0.6 & 10.1 & 0.72 & F & NGC 6218 & -2.3 & 3.5 & 0.6 & 10.1 & 0.60 & F \\
\hline NGC 6712 & -2.3 & 3.7 & 0.8 & 10.1 & 0.77 & $\mathrm{~F}$ & NGC 6838 & -2.3 & 3.2 & 0.55 & 10.1 & 0.78 & $\mathrm{~F}$ \\
\hline
\end{tabular}

Notes: Units for the characteristic mass $m_{\mathrm{c}}$ are $\mathrm{M}_{\odot}$, the age $\log t$ is in $\mathrm{yr}$, while $t_{\mathrm{dyn}}$ is the ratio of the cluster age and the time to dissolution. An age of $12.7 \mathrm{Gyr}$ is assumed for all GCs. For bibliographic references (Ref) see the corresponding letters in the References.

high-quality observations of about a dozen star-forming regions and associations of various ages from the most recent literature, as indicated in Table 1.

To prevent biases in the result, the data should refer to the global MF (GMF), i.e., that of the cluster as a whole. For GCs, where complete cluster coverage is not always possible, we use information on mass stratification and mass segregation to derive the GMF from the local MF (see, e.g., De Marchi et al. 2006). Alternatively, we use the MF measured near the half-light radius, since it has been shown to reflect quite reliably the properties of the GMF (De Marchi et al. 2000). For YCs, we specifically selected those objects for which the coverage is as complete as possible.

The values of the TPL parameters $\left(\alpha, \beta, m_{\mathrm{c}}\right)$ that best fit the data are given in Table 1. Since the GC data do not constrain the mass range $>0.8 \mathrm{M}_{\odot}$, we imposed $\alpha \equiv 2.3$, in line with the average value for YCs. The typical uncertainties in $\alpha$ and $\beta$ are, respectively, 0.1 and 0.2 , while that associated with $m_{\mathrm{c}}$ is $0.05 \mathrm{M}_{\odot}$.

\section{Evolution of the mass function}

Despite the widely different physical properties of the clusters in our sample, e.g., in terms of chemical composition, total mass, stellar density and age, the parameters $\alpha$ and $\beta$ span a relatively narrow range of values, with no significant differences between YCs and GCs: for the former, $\alpha=-2.1 \pm 0.2$ and $\beta=2.4 \pm 0.4$, while for the latter, $\alpha \equiv-2.3$ and $\beta=2.6 \pm 0.3$. The characteristic mass shows a much wider spread. The average value of $m_{\mathrm{c}}$ for dense globular clusters is $0.34 \pm 0.04 \mathrm{M}_{\odot}$ and for loose globulars it grows to $\sim 0.64 \pm 0.10 \mathrm{M}_{\odot}$, although the statistics are limited in this latter case. The variation in $m_{\mathrm{c}}$ is even more pronounced for YCs, ranging from 0.15 to $0.6 \mathrm{M}_{\odot}$. In principle, this variation could reflect differences in the star-formation process, possibly induced by differences in environmental conditions. However, there does not seem to be a correlation between $m_{\mathrm{c}}$ and the clusters' physical properties such as total mass, concentration or metallicity. For example, the dense GCs in our sample span almost two decades in metallicity and total mass, but have the same $m_{\mathrm{c}}$, while all YCs have practically the same metallicity but a wide range of $m_{\mathrm{c}}$ values. Therefore, the environment does not seem to play a role in the shape of the MF of the clusters in our sample.

Another possibility to consider to explain the observed spread in $m_{\mathrm{c}}$ is the role of dynamical evolution, which could alter the shape of the MF over time because of the 
preferential loss of low-mass stars caused by two-body relaxation. It is thus worth investigating whether our data show any correlation or trend between the cluster's characteristic mass and dynamical state. For this reason, we list in Table 1 the dynamical age $t_{\mathrm{dyn}}$ of each cluster, defined as $t / t_{\mathrm{dis}}$ or the ratio of the cluster age and the time to dissolution $t_{\text {dis }}$, i.e., the time at which the cluster has lost $95 \%$ of its original mass. Using $N$-body models, Baumgardt \& Makino (2003) showed that $t_{\mathrm{dis}} \propto t_{\mathrm{rh}}^{x} t_{\mathrm{cr}}^{1-x}$ where $t_{\mathrm{rh}}$ is the halfmass relaxation time, $t_{\mathrm{cr}}$ the crossing time and $x$ depends on the initial concentration of the cluster. Gieles et al. (2005), using the same $N$-body models, found that $t_{\text {dis }}$ can be expressed as a function of the total initial mass of the cluster $M_{\mathrm{ini}}$, as $t_{\mathrm{dis}} \propto t_{0} M_{\mathrm{ini}}^{0.62}$ (see also Gieles, these proceedings), where $t_{0}$ is a constant that depends on the properties of the galaxy and on the cluster orbit. The values of $t_{\mathrm{dyn}}$ in Table 1 come from Baumgardt et al. (2008) for the specific GCs in our sample. For YCs, we have estimated their initial mass and the corresponding value of $t_{\text {dis }}$ following Baumgardt \& Makino $(2003$; in particular their figures 2 and 3) from the current mass given by Piskunov et al. (2008). For clusters younger than $100 \mathrm{Myr}$ we have assumed $t_{\mathrm{dis}} \simeq 500 \mathrm{Myr}$ but, as we will see later, our conclusions would not change if $t_{\text {dis }}$ were larger or smaller by a factor of two (hereafter we will assume an uncertainty of $1 / 3$ on $t_{\mathrm{dis}}$ ). The case of Taurus, however, is different: Ballesteros Paredes et al. (2009) conducted a gravitational analysis of its orbit and concluded that the Taurus molecular cloud must be suffering significant tidal disruption, in spite of its young age $(1 \mathrm{Myr})$. We conservatively assigned to it $t_{\mathrm{dyn}}=1 / 4$, although the actual value could be larger.

The run of $m_{\mathrm{c}}$ as a function of $t_{\mathrm{dyn}}$ is shown in Figure 1, where all clusters are labeled individually, except for the GCs (see caption for details). The figure reveals a rather remarkable trend of increasing $m_{\mathrm{c}}$ with dynamical age over the entire range covered by the data. The dashed line is a purely empirical eyeball fit to the data of the relationship

$$
m_{\mathrm{c}} / \mathrm{M}_{\odot} \simeq 0.15+0.5 \times t_{\mathrm{dyn}}^{3 / 4},
$$

although an index of $1 / 2$ would still provide an acceptable fit. The residual scatter seen in Figure 1 is dominated by two effects: the uncertainty in $t_{\mathrm{dyn}}$ and incomplete coverage of the clusters. As an example of the latter, we show in Figure 1 also the data point corresponding to only the central regions of IC 348 (from the MF of Muench et al. 2003). In spite of the young age of the cluster, the MF in its core shows strong signs of mass segregation, with $m_{\mathrm{c}}=0.33 \mathrm{M}_{\odot}$ as opposed to $m_{\mathrm{c}}=0.14 \mathrm{M}_{\odot}$ for the cluster as a whole. This can be due to primordial mass segregation and/or to very fast dynamical evolution (see, e.g., Allison et al. 2009), but in either case this data point should be excluded from the sample so as not to skew the results. In spite of our attempts to select only clusters with complete radial coverage, however, this information might not be completely available or fully correct in the literature, and this is likely to account for some of the residual scatter that we observe.

\section{Discussion and conclusions}

At least qualitatively, the trend seen in Figure 1 is consistent with the onset of mass segregation through two-body relaxation in a tidal environment, causing the preferential loss of low-mass stars from the cluster and hence a drift of the characteristic mass towards higher values. Vesperini \& Heggie (1997) showed (using $N$-body models) that stellar evaporation, integrated over the cluster's orbit and further enhanced by the presence of the Galactic tidal field, causes a flattening of the MF, i.e., a selective depletion at the low-mass end. Their models do not reveal a drift of characteristic mass towards higher values, but their IMF in all cases is a pure power law. A drift of $m_{\mathrm{c}}$ is visible in the 


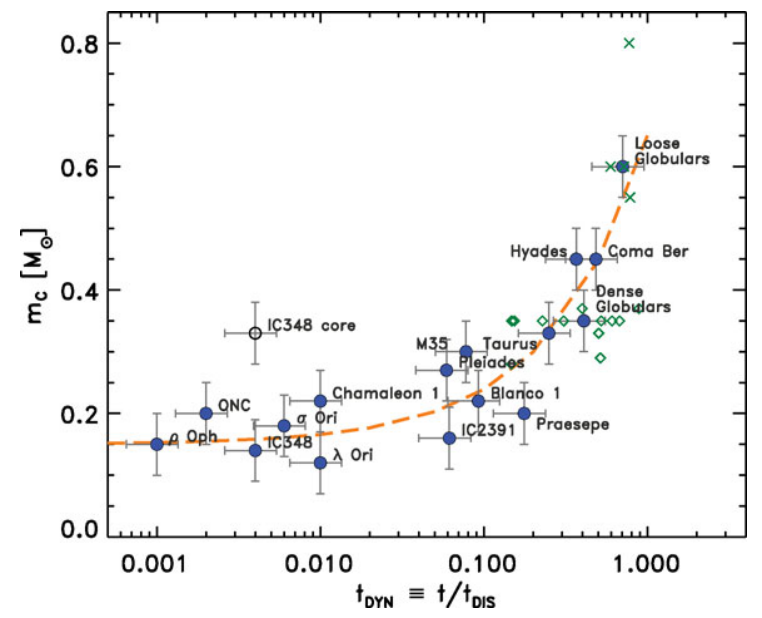

Figure 1. Run of the characteristic mass, $m_{\mathrm{c}}$, as a function of dynamical age. Dense and loose GCs are indicated, respectively, with diamonds and crosses, with a filled circle providing the average value for each class. The core of IC 348 is indicated as an open circle to distinguish it from the whole cluster. The dashed line is an eyeball fit to the data.

$N$-body models of Portegies Zwart et al. (2001), but it proceeds very slowly and only appears when the study of the MF is limited to the regions inside the half-mass radius. On the other hand, De Marchi et al. (2007) showed that dense and loose GCs have today systematically different GMFs, consistent with a much stronger selective loss of low-mass stars in the latter. The fact that $m_{\mathrm{c}}$ is systematically higher in loose GCs proves that its value must drift as a result of the cluster's dynamical evolution, even though the analysis of the $N$-body model results has so far failed to detect it.

An important consequence of the trend shown in Figure 1, if dynamical evolution is indeed at its origin, is that it seems then perfectly plausible that all GCs, having now $m_{\mathrm{c}} \simeq 0.35 \mathrm{M}_{\odot}$ if they are dense or $\sim 0.65 \mathrm{M}_{\odot}$ if they are loose, were born with a stellar MF like that measured today in the youngest Galactic clusters, with $m_{\mathrm{c}} \simeq 0.15 \mathrm{M}_{\odot}$.

This conclusion is fully consistent with the absence of a turnover in the MF of the Galactic bulge down to the observational limit at $\sim 0.2 \mathrm{M}_{\odot}$ (Zoccali et al. 2000 ). Regardless as to whether the bulge is primarily the result of fast formation at early epochs (e.g., Ballero et al. 2007) or a collection of the stars lost from disrupted clusters throughout the life of the Galaxy (e.g., Gnedin \& Ostriker 1997), its very location at the bottom of the Galaxy's potential well makes it very hard for low-mass stars to escape. Therefore, its present MF should not have been altered by dynamical evolution, unlike the case of the comparably old GCs, and should still reflect the properties of the IMF. Forthcoming HST observations of the Galactic bulge (Brown et al. 2009) will provide insights into the MF of the bulge below $0.15 \mathrm{M}_{\odot}$, where we expect a turnover.

If the picture sketched above is correct and dynamical evolution is the main source of MF variations in Galactic clusters, it would argue quite strongly in favour of the universality of the IMF of Population I and II stars. Previous claims in this sense (e.g., Gilmore 2001; Elmegreen et al. 2008) were based on the observation that the stellar MFs in different environments have similar $m_{\mathrm{c}}$ values, within factors of a few. Here, we show for the first time that the value of $m_{\mathrm{c}}$ may in fact be exactly the same, suggesting that environmental conditions do not play a significant role in the process of star formation, or at least regarding its end result. Compelling theories have already been presented as to why this should be the case (Bate 2009; Goodwin \& Kouwenhoven 2009). 


\section{Acknowledgements}

SPZ acknowledges the support of NWO through grant \# 639.073.803.

\section{References}

Allison, R., Goodwin, S. P., Parker, R. J., de Grijs, R., Portegies Zwart, S. F., \& Kouwenhoven, M. B. N. 2009, ApJ (Letters), 700, L99

Ballesteros-Paredes, J., Gómez, G. C., Loinard, L., Torres, R. M., \& Pichardo, B. 2009, MNRAS (Letters), 395, L81

Ballero, S., Matteucci, F., Origlia, L., \& Rich, M. 2007, A\&\&A, 467, 123

Barrado y Navascués, D., Stauffer, J. R., Bouvier, J., Jayawardhana, R., \& Cuillandre, J.-C. 2004, ApJ, 610, 1064 (A)

Barrado y Bavascués, D., Stauffer, J. R., \& Jayawardhana, R. 2004, ApJ, 614, 386 (B)

Bate, M. 2009, MNRAS, 397, 232

Baumgardt, H., De Marchi. G., \& Kroupa, P. 2008, ApJ, 685, 247

Baumgardt, H. \& Makino, J. 2003, MNRAS, 340, 227

Bouvier, J., et al. 2008, A\&A 481, 661 (C)

Brown, T., et al. 2009, AJ, 137, 3172

Caballero, J. 2008, A\&A A, 478, 667 (D)

Chabrier, G. 2003, PASP, 115, 763

Chappelle, R., Pinfield, D. J., Steele, I. A., Dobbie, P. D., \& Magazzù, A. 2005, MNRAS, 361, $1323(\mathrm{E})$

De Marchi, G., Paresce, F., \& Portegies Zwart, S. 2005, in: E. Corbelli, F. Palla, \& H. Zinnecker (eds.), The Initial Mass Function 50 years later (Dordrecht: Springer), p. 77

De Marchi, G., Paresce, F., \& Pulone, L. 2000, ApJ, 530, 342

De Marchi, G., Paresce, F., \& Pulone, L. 2007, ApJ (Letters), 656, L65 (F)

De Marchi, G., Pulone, L., \& Paresce, F. 2006, A\&A, 449, 161

Elmegreen, B., Klessen, R., \& Wilson, C. 2008, ApJ, 681, 365

Gieles, M., Bastian, N., Lamers, H. J. G. L. M., \& Mout, J. N. 2005, A\&\&A, 441, 949

Gilmore, G. 2001, in: L. Tacconi \& D. Lutz (eds.), Starburst Galaxies (Heidelberg: Springer), p. 34

Gnedin, O. \& Ostriker, J. 1997, ApJ, 474, 223

Goodwin, S. \& Kouwenhoven, M. 2009, MNRAS (Letters), 397, L36

Kraus, A. \& Hillenbrand, L. 2007, AJ, 134, 2340 (G)

Kroupa, P. 2002, in: E. Grebel \& W. Brandner (eds.), Modes of Star Formation and the Origin of Field Populations, ASP Conf. Ser. 285, (San Francisco: ASP), p. 86

Luhman, K. 2007, ApJS, 173, $104(\mathrm{H})$

Luhman, K. \& Rieke, G. 1999, ApJ, 525, 440 (I)

Moraux, E., Bouvier, J., Stauffer, J. R., Barrado y Navascués, D., \& Cuillandre, J.-C. 2007, $A \mathscr{E} A, 471,499(\mathrm{~J})$

Muench, A., Lada, E. A., Lada, C. J., Elston, R. J., Alves, J. F., Horrobin, M., Huard, T. H., Levine, J. L., Raines, S. N., \& Román-Zúñiga, C. 2003, AJ, 125, 2029 (K)

Paresce, F. \& De Marchi, G. 2000, ApJ, 534, 870 (L)

Piskunov, A., Schilbach, E., Kharchenko, N. V., Röser, S., \& Scholz, R.-D. 2008, A\& A, 477, 165

Portegies Zwart, S., McMillan, S. L. W., Hut, P., \& Makino, J. 2001, MNRAS, 321, 199

Salpeter, E. 1955, ApJ, 121, 161

Slesnick, C., Hillenbrand, L. A., \& Carpenter, J. M. 2004, ApJ, 610, 1045 (M)

Spitzer, L. 1987, Dynamical evolution of globular clusters, (Princeton: Princeton University Press)

Vesperini, E. \& Heggie, D. 1997, MNRAS, 289, 898

Zoccali, M., Cassisi, S., Frogel, J. A.; Gould, A., Ortolani, S., Renzini, A., Rich, R. M., \& Stephens, A. W. 2000, ApJ, 530, 418 